Original research article

\title{
Evaluation of recanalisation treatment on posterior circulation ischemic stroke by Solitaire device-A multicenter retrospective study
}

\author{
Jianwu Long ${ }^{a, b}$, Hao Qin ${ }^{c}$, Hongqi Zhang ${ }^{a, *}$ \\ a Department of Neurosurgery, Xuanwu Hospital, Capital Medical University, Beijing, PR China \\ b Department of Neurosurgery, The 3rd Hospital of Xiamen, Fujian, PR China \\ ${ }^{\mathrm{c}}$ Department of Neurosurgery, Zaozhuang Municipal Hospital, Shandong, PR China
}

\section{A R T I C L E I N F O}

\section{Article history:}

Received 29 September 2016

Accepted 23 February 2017

Available online 24 March 2017

Keywords:

Posterior circulation ischemic stroke Endovascular mechanical

thrombectomy

Multicenter retrospective study

Solitaire device

Artery recanalization

\begin{abstract}
A B S T R A C T
Objectives: Posterior circulation ischemic stroke (PCIS), accounting for approximately $20 \%$ of total ischemic stroke, is a sever disease that associated with high rate of morbidity and mortality. Though the effectiveness of endovascular mechanical thrombectomy has been well demonstrated in many types of ischemic stroke, it is still unclear what the outcome is in posterior circulation ischemic stroke.

Methods and materials: In current study, data was collected from 139 Chinese patients who received endovascular mechanical thrombectomy treatment with Solitaire device after acute posterior circulation ischemic stroke. We measured the mortality, symptomatic intracranial hemorrhage (SICH) and National Institutes of Health Stroke Scale (NIHSS) to evaluate the safety of endovascular mechanical thrombectomy. Meanwhile, the clinical outcome of endovascular mechanical thrombectomy was also evaluated based on recanalisation rate, HIHSS, and the modified Rankin Scale (mRS).

Results: Recanalisation was successful in 124 (89.3\%) patients after surgery. Herniation was the second fatal stroke complication, out of the 6 patients suffered from herniation, 3 patients (50\%) died during surgery and 2 (33\%) died after surgery. As for other stroke complications such as pulmonary infection, 1 patient $(4.3 \%)$ died during surgery and 1 patient (4.3\%) died 3 days after surgery.

Conclusion: Our findings indicate that endovascular mechanical treatment is a safe treatment which brings clear benefit to patients suffered from posterior circulation ischemic stroke, in both the recanalisation rate and functional outcomes.
\end{abstract}

(c) 2017 Published by Elsevier Sp. z o.o. on behalf of Polish Neurological Society.

\footnotetext{
* Corresponding author at: Department of Neurosurgery, Xuanwu Hospital, Capital Medical University, No. 45 Changchun Street, Beijing, PR China.

E-mail address: hqzh@vip.163.com (H. Zhang).

http://dx.doi.org/10.1016/j.pjnns.2017.02.004

0028-3843/@ 2017 Published by Elsevier Sp. z o.o. on behalf of Polish Neurological Society.
} 


\section{Introduction}

Acute basilar artery occlusion (BAO) caused the most severe types of ischemic stroke, without active treatment, the mortality rate can be up to $90 \%$ [1,2]. Anterior and posterior circulations were two important artery systems supplied by basilar artery. Based on the location where infarction occurred within the vascular territory, BAO could lead to anterior or posterior circulation ischemic stroke [1,3]. The two differs from both stroke etiology [4,5] and in terms of morbidity and mortality rate [6].

Although rarer than anterior circulation ischemic stroke (ACIS), posterior circulation ischemic stroke (PCIS) accounts for approximately $20 \%$ of all ischemic stroke [1,7-9], and without proper treatment, it might leads to potentially preventable death or severe disability [10].

Thrombolysis is an effective medical therapy for PCIS patients, however, with a strictly limited time window. Based on series of randomized large sample clinical studies, intravenous tissue-type plasminogen activator (tPA) must be applied within up $4.5 \mathrm{~h}$ of symptom onset [11-13].

Multiple recent studies showed consistent results that endovascular mechanical thrombectomy, a technique which clears local thrombosis by mechanical methods, can bring a clear benefit to acute anterior circulation ischemic stroke (ACIS) patients, with a prolonged treatment window [14-18]. Moreover, the combination of tPA treatment with endovascular mechanical thrombectomy had largely facilitated artery recanalisation of ischemic stroke patients $[14,19,20]$. Most of the studies were based on data from single center or a limited number of patients with acute ACIS [21-26]. PCIS differs from ACIS in terms of both stroke etiology $[4,25]$ and higher rate of morbidity and mortality [6]. Evidence is needed to evaluate the clinical outcomes of endovascular mechanical thrombectomy on PCIS patients.

We undertook the current multicenter, retrospective study with large sample size on the effectiveness of endovascular mechanical thrombectomy on PCIS patients. We collected the data of 139 Chinese patients suffering from posterior circulation stroke in the database of Acute Cerebrovascular Disease (http://www.acute-cvd.com), analyzed and evaluated the clinical outcomes of these patients treated within $24 \mathrm{~h}$ after stroke onset. Both the safety and efficacy of endovascular mechanical thrombectomy with Solitare device, one of the most effective recanalisation devices [27,28], on these patients, and the related clinical prognostic factors 90 days after treatment were analyzed.

\section{Methods}

\subsection{Patient population}

We collected data of 139 Chinese patients suffered acute ischemic stroke and received hospitalization in multiple centers located in China from January 2012 to December 2015. All data were acquired from the database of Acute Cerebrovascular Disease (http://www.acute-cvd.com). From the total 643 patients in the database, with either anterior (500 cases) or posterior (140 cases) circulation ischemic stroke, we selected patients based on the following criteria: (1) diagnosed as acute posterior circulation ischemic stroke, with Digital Subtraction Angiography (DSA) to confirm the location of occlusion; (2) aging from 18 to 80 years old; (3) baseline National Institutes of Health Stroke Scale (NIHSS) $\geq 8$; (4) Thrombolysis In Cerebral Infarction (TICI) is between 0 and 1, a criteria proposed by Solitaire device for patients to receive endovascular mechanical thrombectomy, assessed by Computed Tomography Angiography (CTA), Magnetic Resonance Angiography (MRA) or Digital Subtraction Angiography (DSA); (5) accumulated Alberta Stroke Program Early CT Score (ASPECTS) $\geq 6$ and the pons-mesencephalon score $0-2$ (see Fig. 1); (6) received endovascular treatment within $24 \mathrm{~h}$ after stroke onset. Although most patients suffered only basilar artery occlusion, there were some cases where vertebral artery or the posterior cerebral artery occlusion was observed.

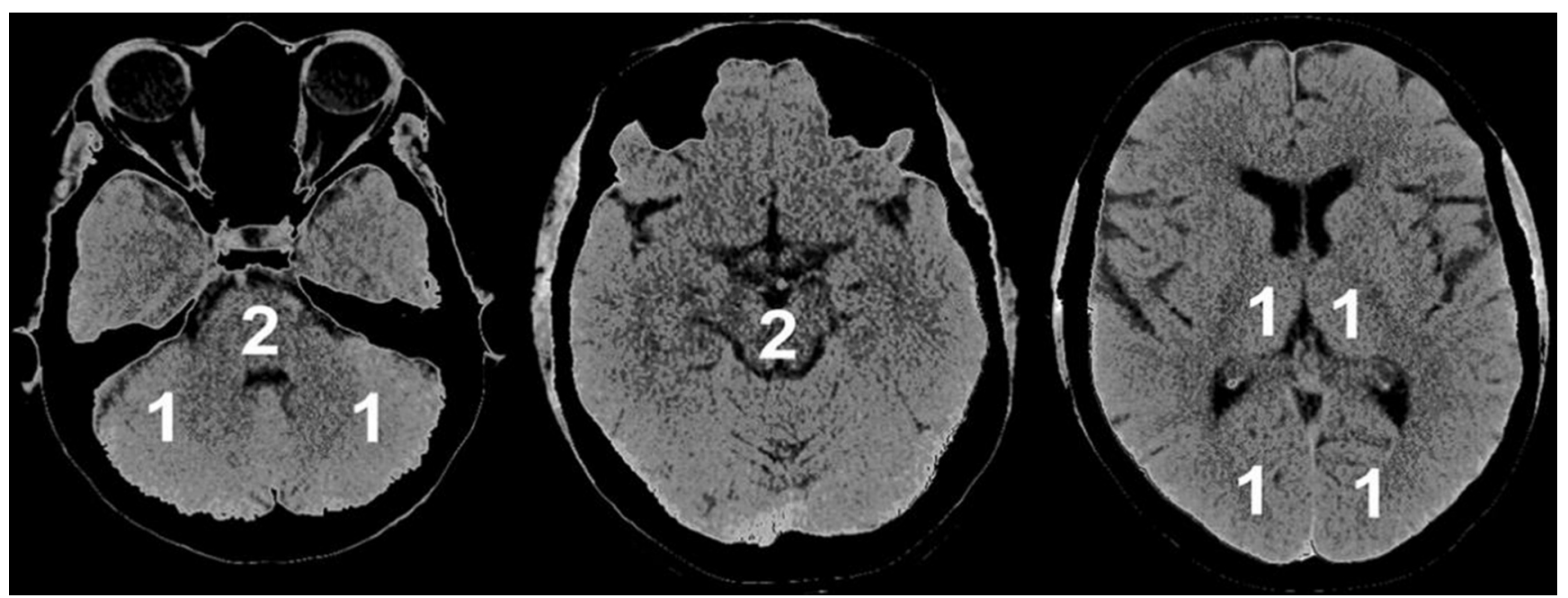

Fig. 1 - pc-ASPECTS score: the three figures showed examples of pons-mesencephalon score from 0 to 2 , as indicated in the figures. 


\subsection{Endovascular thrombectomy with Solitaire}

All the patients received endovascular mechanical thrombectomy treatment under anesthesia. The surgery procedure includes following steps.

After femoral artery puncture, whole-brain vessels angiography was performed to locate the blocked artery and to confirm the perfusion state of its neighboring vessels (TICI scales). A microcatheter was penetrated through the thrombus to perform superselectire angiography, in order to confirm the length of the thrombus and the perfusion state of the vessel distal to the thrombus.

To perform the endovascular mechanical thrombectomy, an 8-F guiding catheter was located at the proximal end of the thrombus through transfemoral access. Then a Rebar18 microcatheter was carefully navigated through the thrombus over a 0.014-in. microwire to reach the distal end of the thrombus (Fig. 2, up left). The end of the stent retriever was left several millimeters farther than the distal end of the thrombus. Then the microcatheter was withdrawn back to the proximal end of the thrombus and the stent retriever was allowed to fully expand all through the thrombus (Fig. 2, up right). While keeping its fully expanded status, the stent retriever was carefully pulled back to recanalise the occluded artery (Fig. 2, down left). The end of the guiding catheter was opened and the thrombus fragments along with the stent retriever were sucked out by a $50 \mathrm{ml}$ syringe (Fig. 2, down right). Imaging was followed immediately to confirm the artery recanalisation.

After surgery, the patients were administered with a dual dose of $75 \mathrm{mg}$ clopidogrel and $100 \mathrm{mg}$ aspirin for antiplatelet treatment.

\subsection{Variables of interest}

\subsubsection{Safety evaluation}

The safety of endovascular mechanical thrombectomy was evaluated by the following variables: (1) mortality rate 90 days
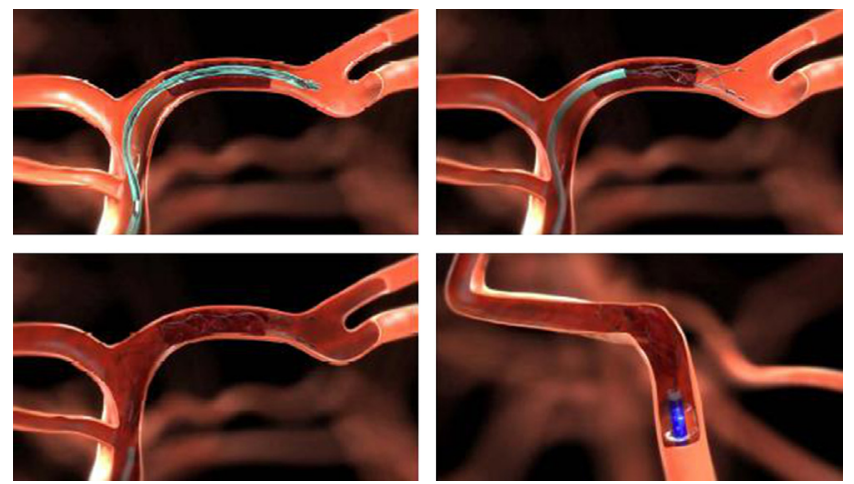

Fig. 2 - Endovascular mechanical thrombectomy by Solitaire device. Up left: 0.014-in. microwire to reach the distal end of the thrombus. Up right: microcatheter withdrawn back to the proximal end of the thrombus and the stent retriever fully expand all through the thrombus. Down left: stent retriever was carefully pulled back to recanalise the occluded artery. Down right: guiding catheter end opened and the thrombus fragments sucked out by a $50 \mathrm{ml}$ syringe. after surgery; (2) the rate of symptomatic intracranial hemorrhage (SICH). The judgment of SICH was based on the evidence of CT or MRI. SICH was defined as the intracranial hemorrhage within 22-36 h after treatment as confirmed by imaging techniques, accompanied with NIHSS decrease $\geq 4$.

\subsubsection{Effectiveness evaluation}

The effectiveness of endovascular mechanical thrombectomy was evaluated as the recanalisation rate, represented by TICI ( 2 or 3). Besides, the NIHSS score at discharge and 90 days after endovascular mechanical thrombectomy surgery were analyzed. Modified Rankin Scale (mRS) 90 days post surgery was measured. The complications such as symptomatic intracerebral hemorrhage, vessel dissection and the occlusion of unaffected vessels were monitored as well.

\subsubsection{Factors affecting clinical outcomes}

We monitored the factors that could potentially affect clinical outcomes, including (1) factors related with patients: (i) age, (ii) baseline blood pressure, (iii) diabetes, (iv) NIHSS or GCS before treatment; (2) factors related with endovascular mechanical thrombectomy: (i) anesthesia, (ii) number of endovascular mechanical thrombectomy received, (iii) length of time from arriving hospital to recanalisation, (iv) type of stroke (TOAST classification), (v) combined treatment regime, (vi) symptomatic hemorrhage.

\subsection{Statistical analysis}

Descriptive analysis on frequencies or percentages of classified variables was performed. Chi-square test was performed on continuous variables that fit normal distribution. For the continuous variables that did not fit normal distribution, median and quartile values were used for analysis. To compare patients with favorable vs. unfavorable prognosis, t-test or rank sum test was performed on the continuous variables, while chi-square test or Fisher exact probability test was performed on classified variables. One-way analysis of variance (ANOVA) was performed on factors predicting the change of clinical phase. All the analysis was performed on software SPSS 19.0.

\section{Results}

\subsection{Patient data}

In this study, we collected data of 139 Chinese patients suffered acute ischemic stroke and received endovascular mechanical recanalisation from the database of Acute Cerebrovascular Disease (http://www.acute-cvd.com). In our collection, there were 105 male and 34 female patients, with an average age of $59.7 \pm 13.6$. The median pre-thrombectomy NIHSS score was 23 (IQR: 16-28) and the median pre-thrombectomy GCS was 9 (IQR: 7-10) (Table 1).

\subsection{Treatment results and clinical outcomes}

In current dataset, the median onset-to-door time was 310 (IQR: 240-498) min and the median surgery time was 72 (IQR: 


\section{Table 1 - Patient characteristics.}

\section{Characteristics}

Number of patients

Male (\%)

Age (year), mean (SD)

Pre-thrombectomy NIHSS, median (IQR)

Pre-thrombectomy GCS, median (IQR)

50-120) min. 14 patients died during the surgery (mortality rate: $10 \%)$ and the mortality rate reached $14.3 \%$ (20/139) by the time of 90 days after surgery. Favorable outcomes (mRS scores: $0-2)$ were achieved in 34 patients at discharge $(24.5 \%$ of all the patients, $27.2 \%$ of the survivors). By the time of 90 days after surgery, favorable outcomes (mRS scores: 0-2) were achieved in 58 patients $(41.7 \%$ of all the patients, $48.7 \%$ of the survivors).

The patients' median post-thrombectomy NIHSS score was 17.0 (IQR: 7.5-24.5) at discharge after endovascular mechanical thrombectomy surgery. Recanalisation was successful in 124 patients (89.2\%) with TICI grades $\geq 2 \mathrm{~b}$; furthermore, $62.1 \%$ of these patients achieved TICI grade 3 . By the time of 90 days after surgery, the median NIHSS score was 10.0 (IQR: 4.8-20.0) (Table 2). Endovascular mechanical thrombectomy surgery with Solitaire device had improved NIHSS score significantly, both at discharge $(P<0.0001)$ and 90 days after surgery $(P<0.0001)$.

However, seven kinds of stroke complications were observed in the patients during or after surgery: 23 patients $(16.5 \%)$ suffered from pulmonary infection, 5 patients $(3.6 \%)$ suffered from hemorrhagic transformation, 6 patients $(4.3 \%)$ suffered from herniation, 1 patient $(0.7 \%)$ suffered from hydrocephalus, 1 patient $(0.7 \%)$ suffered from gastrointestinal hemorrhage, 4 patients suffered from cardiac or respiratory arrest $(2.9 \%)$ and 1 patient $(0.7 \%)$ suffered from recurrent stroke (Table 2).

\begin{tabular}{ll} 
Table 2 - Treatment outcomes. & \\
Characteristics & \multicolumn{1}{c}{ Value } \\
\hline Onset-to-door time (min), median (IQR) & $310(240-498)$ \\
Surgery time (min), median (IQR) & $72(50-120)$ \\
TICI after surgery, $n$ (\% of all, \% of survivors) & \\
$\quad \geq 2 \mathrm{~b}$ & $124(89.2,99.2)$ \\
3 & $77(55.4,61.6)$ \\
NIHSS at discharge, median (IQR) & $17.0(7.5-24.5)$ \\
mRS at discharge, $n$ (\% of all, \% of survivors) & \\
0-2 & $34(24.5,27.2)$ \\
Mortality at discharge, $n$ (\%) & $14(10)$ \\
NIHSS 90 days after surgery, median (IQR) & $10.0(4.8-20.0)$ \\
mRS 90 days after surgery, $n$ & \\
(\% of all, \% of survivors) & \\
0-2 & $58(41.7,48.7)$ \\
Mortality 90 days after surgery, $n$ (\%) & $20(14.4)$ \\
Stroke complications, $n$ (\%) & \\
Pulmonary infection & $23(16.5)$ \\
Hemorrhagic transformation & $5(3.6)$ \\
Herniation & $6(4.3)$ \\
Hydrocephalus & $1(0.7)$ \\
Gastrointestinal hemorrhage & $1(0.7)$ \\
Cardiac or respiratory arrest & $4(2.9)$ \\
Recurrent stroke & $1(0.7)$ \\
\hline & \\
\hline
\end{tabular}

\section{Discussion}

Endovascular mechanical thrombectomy with a stent retriever was now the most recommended treatment in patients suffered from BAO of anterior cerebral circulation [14-18]. However, on the other hand, there were only a few small sample studies focusing on the safety and effectiveness of endovascular mechanical thrombectomy treatment on patients suffered from acute posterior circulation ischemic stroke, another important stroke type caused by BAO [22,2932]. To the best of our knowledge, this study was the first multi-center study with large sample size to evaluate the safety and effectiveness of endovascular mechanical thrombectomy treatment with Solitaire device on acute posterior circulation ischemic stroke on 139 Chinese patients.

Our study showed improved outcomes of endovascular mechanical thrombectomy treatment with Solitaire device on patients suffered from acute posterior circulation ischemic stroke than reported results with either traditional treatment [2] or thrombolysis therapy [33,34] in the following aspects: reduced mortality rate, higher recanalisation rate and better clinical outcomes. As compared with traditional treatment, our mechanical thrombectomy treatment with Solitaire device showed a much lower mortality rate (10\% after surgery and $14.3 \%$ by 90 days vs. traditional $40 \%$ ) as well as improved outcomes (34\% at mRS 0-2 vs. traditional 17\% at mRS 0-3) [2]. By 90 days after treatment, our method showed an even better outcome (58\% at $\mathrm{mRS} 0-2)$ than that with traditional treatment. As compared with another widely used treatment for ischemic stroke, thrombolysis therapy, our method achieved a higher recanalisation rate (89.2\% vs. $53-65 \%)$ $[33,34]$. All these results suggest that mechanical thrombectomy with Solitaire device could serve as a safe and efficient treatment for patients suffered from acute posterior circulation ischemic stroke, which could provide better outcomes than traditional treatment or thrombolysis therapy. Furthermore, even when compared with mechanical thrombectomy treatment with other devices, such as Merci device, our mortality rate with Solitaire device was much lower (10\% after surgery and $14.3 \%$ by 90 days vs. $43-50 \%$ with Merci) along with a much shorter operation time (73 with Solitaire vs. 129 min with Merci) [40]. Our operation time was comparable to a recent single center study with Solitaire device (73 vs. $61 \mathrm{~min}$ ), but with a lower mortality rate (14.3 vs. $32 \%$ ) [22]. These results suggest that both our treatment strategy and operation techniques are competent to the best level of mechanical thrombectomy.

In current study, stroke complications during and after surgery have a strong correlation with after surgery mortality (65\% of the 20 deaths). Cardiac or respiratory arrest was the most fatal stroke complication and all four patients suffered from it died during surgery (100\%). Herniation was the second fatal stroke complication, out of the six patients suffered from herniation, three (50\%) died during surgery and two (33\%) died after surgery. As for other stroke complications such as pulmonary infection, one patient $(4.3 \%)$ died during surgery and one patient $(4.3 \%)$ died three days after surgery. Two patients suffered from hemorrhagic transformation (40\%) died during surgery. Developing strategies to monitor and reduce 
stroke complications is a potential target to further improve the safety and efficacy of mechanical thrombectomy treatment on acute posterior circulation ischemic stroke patients.

\section{Conflict of interest}

None declared.

\section{Acknowledgment and financial support}

This study was supported by National Tech \& Sci Supporting Plan Fund (No. 2011BAI08B00).

\section{Ethics}

The work described in this article has been carried out in accordance with The Code of Ethics of the World Medical Association (Declaration of Helsinki) for experiments involving humans; Uniform Requirements for manuscripts submitted to Biomedical journals.

\section{R E F E R E N C E S}

[1] Mattle HP, Arnold M, Lindsberg PJ, Schonewille WJ, Schroth G. Basilar artery occlusion. Lancet Neurol 2011;10:1002-14.

[2] Schonewille WJ, Algra A, Serena J, Molina CA, Kappelle LJ. Outcome in patients with basilar artery occlusion treated conventionally. J Neurol Neurosurg Psychiatry 2005;76:1238-41.

[3] Tatu L, Moulin T, Bogousslavsky J, Duvernoy H. Arterial territories of human brain: brainstem and cerebellum. Neurology 1996;47:1125-35.

[4] Caplan LR. Posterior circulation disease: clinical findings, diagnosis and management. Cambridge, MA: Science Inc.; 1996.

[5] Bogousslavsky J, Regli F, Maeder P, Meuli R, Nader J. The etiology of posterior circulation infarcts: a prospective study using magnetic resonance imaging and magnetic resonance angiography. Neurology 1993;43:1528-33.

[6] Hornig CR, Buttner T, Hoffmann O, Dorndorf W. Short-term prognosis of vertebrobasilar ischemic stroke. Cerebrovasc Dis 1992;2:273-81.

[7] Nouh A, Remke J, Ruland S. Ischemic posterior circulation stroke: a review of anatomy, clinical presentations, diagnosis, and current management. Front Neurol 2014;5:30.

[8] Gulli G, Marquardt L, Rothwell PM, Markus HS. Stroke risk after posterior circulation stroke/transient ischemic attack and its relationship to site of vertebrobasilar stenosis: pooled data analysis from prospective studies. Stroke 2013;44:598-604.

[9] Labropoulos N, Nandivada P, Bekelis K. Stroke of the posterior cerebral circulation. Int Angiol 2011;30:105-14.

[10] Kuruvilla A, Bhattacharya P, Rajamani K, Chaturvedi S. Factors associated with misdiagnosis of acute stroke in young adults. J Stroke Cerebrovasc Dis 2011;20:523-7.

[11] Hacke W, Kaste M, Bluhmki E, Brozman M, Dávalos A, Guidetti D, et al. Thrombolysis with alteplase 3 to 4.5 hours after acute ischemic stroke. N Engl J Med 2008;359:1317-29. CrossRefMedlineWeb of Science.
[12] Förster A, Gass A, Kern R, Griebe M, Hennerici MG, Szabo K. Thrombolysis in posterior circulation stroke: stroke subtypes and patterns, complications and outcome. Cerebrovasc Dis 2011;32:349-53. CrossRefMedline.

[13] Sarraj A, Medrek S, Albright K, Martin-Schild S, Bibars W, Vahidy F, et al. Posterior circulation stroke is associated with prolonged door-to-needle time. Int J Stroke 2013. published online 22 March.

[14] Saver JL, Goyal M, Bonafe A, Diener HC, Levy EI, Pereira VM, et al. Stent-retriever thrombectomy after intravenous t-PA vs. t-PA alone in stroke. N Engl J Med 2015;372:2285-95.

[15] Berkhemer OA, Fransen PS, Beumer D, van den Berg LA, Lingsma HF, Yoo AJ, et al. A randomized trial of intraarterial treatment for acute ischemic stroke. N Engl J Med 2015;372:11-20.

[16] Campbell BC, Mitchell PJ, Kleinig TJ, Dewey HM, Churilov L, Yassi N, et al. Endovascular therapy for ischemic stroke with perfusion-imaging selection. N Engl J Med 2015;372:1009-18.

[17] Goyal M, Demchuk AM, Menon BK, Eesa M, Rempel JL, Thornton J, et al. Randomized assessment of rapid endovascular treatment of ischemic stroke. N Engl J Med 2015;372:1019-30.

[18] Jovin TG, Chamorro A, Cobo E, de Miquel MA, Molina CA, Rovira A, et al. Thrombectomy within 8 hours after symptom onset in ischemic stroke. N Engl J Med 2015;372:2296-306.

[19] Przybylowski CJ, Ding D, Starke RM, Durst CR, Crowley RW, Liu KC. Evolution of endovascular mechanical thrombectomy for acute ischemic stroke. World J Clin Cases 2014;2:614-22.

[20] Smith WS. Safety of mechanical thrombectomy and intravenous tissue plasminogen activator in acute ischemic stroke. Results of the multi Mechanical Embolus Removal in Cerebral Ischemia (MERCI) trial, part I. AJNR Am J Neuroradiol 2006;27:1177-82.

[21] Huo X, Gao F, Sun X, Ma N, Song L, Mo D, et al. Endovascular mechanical thrombectomy with the solitaire device for the treatment of acute basilar artery occlusion. World Neurosurg 2016;89:301-8.

[22] Mourand I, Machi P, Milhaud D, Picot MC, Lobotesis K, Arquizan C, et al. Mechanical thrombectomy with the Solitaire device in acute basilar artery occlusion. J Neurointervent Surg 2014;6:200-4.

[23] Carneiro AA, Rodrigues JT, Pereira JP, Alves JV, Xavier JA. Mechanical thrombectomy in patients with acute basilar occlusion using stent retrievers. Intervent Neuroradiol 2015;21:710-4.

[24] Gilberti N, Gamba M, Premi E, Costa A, Vergani V, Delrio I, et al. Endovascular mechanical thrombectomy in basilar artery occlusion: variables affecting recanalization and outcome. J Neurol 2016;263:707-13.

[25] Park BS, Kang CW, Kwon HJ, Choi SW, Kim SH, Koh HS, et al. Endovascular mechanical thrombectomy in basilar artery occlusion: initial experience. J Cerebrovasc Endovasc Neurosurg 2013;15:137-44.

[26] Pfefferkorn T, Mayer TE, Opherk C, Peters N, Straube A, Pfister HW, et al. Staged escalation therapy in acute basilar artery occlusion: intravenous thrombolysis and on-demand consecutive endovascular mechanical thrombectomy: preliminary experience in 16 patients. Stroke 2008;39: 1496-500.

[27] Costalat V, Machi P, Lobotesis K, Maldonado I, Vendrell JF, Riquelme C, et al. Rescue, combined, and stand-alone thrombectomy in the management of large vessel occlusion stroke using the solitaire device: a prospective 50patient single-center study: timing, safety, and efficacy. Stroke 2011;42:1929-35. 
[28] Castano C, Dorado L, Guerrero C, Millan M, Gomis M, Perez de la Ossa N, et al. Mechanical thrombectomy with the Solitaire AB device in large artery occlusions of the anterior circulation: a pilot study. Stroke 2010;41:1836-40.

[29] Andersson T, Kuntze Soderqvist A, Soderman M, Holmin S, Wahlgren N, Kaijser M. Mechanical thrombectomy as the primary treatment for acute basilar artery occlusion: experience from 5 years of practice. J Neurointervent Surg 2013;5:221-5.

[30] Roth C, Mielke A, Siekmann R, Ferbert A. First experiences with a new device for mechanical thrombectomy in acute basilar artery occlusion. Cerebrovasc Dis 2011;32:28-34.

[31] Mordasini P, Brekenfeld C, Byrne JV, Fischer U, Arnold M, Heldner MR, et al. Technical feasibility and application of mechanical thrombectomy with the Solitaire FR Revascularization Device in acute basilar artery occlusion. AJNR Am J Neuroradiol 2013;34:159-63.

[32] Espinosa de Rueda M, Parrilla G, Zamarro J, Garcia-Villalba B, Hernandez F, Moreno A. Treatment of acute vertebrobasilar occlusion using thrombectomy with stent retrievers: initial experience with 18 patients. AJNR Am J Neuroradiol 2013;34:1044-8.

[33] Lindsberg PJ, Mattle HP. Therapy of basilar artery occlusion: a systematic analysis comparing intra-arterial and intravenous thrombolysis. Stroke 2006;37:922-8.

[34] Smith WS. Intra-arterial thrombolytic therapy for acute basilar occlusion: pro. Stroke 2007;38:701-3. 